\title{
REPRESENTAÇÕES SOCIAIS DO MEIO AMBIENTE PARA PESSOAS DE DIFERENTES FAIXAS ETÁRIAS
}

\author{
SOCIAL REPRESENTATIONS OF THE ENVIRONMENT FOR PEOPLE OF DIFFERENT AGES \\ REPRESENTACIONES SOCIALES DEL MEDIO AMBIENTE PARA LAS PERSONAS DE DIFERENTES EDADES
}

\author{
Gislei Mocelin Polli* \\ Brigido Vizeu Camargo
}

\begin{abstract}
RESUMO
Há algumas décadas, o modo de pensar o meio ambiente vem passando por mudanças. A visão que o meio ambiente servia para atender às necessidades humanas deu lugar à ideia de que os seres humanos são parte dele e que ele deve ser cuidado. Este estudo buscou identificar atitudes e representaçóes sociais do meio ambiente de pessoas de diferentes faixas etárias. Para tanto, foi utilizada a escala new environmental paradigm (NEP) que mede atitudes em relação ao meio ambiente. Foram investigadas as representações sociais dos respondentes sobre o meio ambiente, por meio da realização de uma análise de contraste pelo programa Alceste. Participaram da pesquisa 150 pessoas, divididas em três faixas etárias. Não foram encontradas diferenças significativas entre os respondentes de diferentes idades, e todos os respondentes parecem estar de acordo com a nova maneira de pensar o meio ambiente, reconhecendo sua fragilidade e a necessidade de cuidado que ele demanda.
\end{abstract}

Palavras-chave: Representações sociais. Meio ambiente. Psicologia social.

\begin{abstract}
Some decades ago, the way of thinking about the environment has undergone changes. The view that the soul function of the environment was to meet human needs has been replaced by the idea that humans are part of it and they should take care of it. This study sought to identify attitudes and social representations of the environment of people of different ages. The NEP scale (New Environmental Paradigm) was used to measure attitudes to the environment. We investigated the social representations of the respondents about the environment, by conducting a contrast analysis using the Alceste.
\end{abstract}

\footnotetext{
Submetido em 10 de junho de 2013 e aprovado para publicação em 15 de junho de 2016.

Doutora em Psicologia pela UFSC; professora adjunta no Mestrado em Psicologia da Universidade Tuiuti do Paraná. Endereço: Rua Nicarágua, 1995, ap. 705 B - Bacacheri, Curitiba-PR, Brasil. CEP: 82515-260. E-mail: gismocelin@gmail.com.

* Presidente da Federação Iberoamericana de Associações de Psicologia (FIAP); professor associado do Departamento de Psicologia; coordenador do Laboratório de Psicologia Social da Comunicação e Cognição Social (Laccos), Universidade Federal de Santa Catarina. Endereço: Universidade Federal de Santa Catarina, Centro de Filosofia e Ciências Humanas, Departamento de Psicologia, Campus Universitário Trindade, Bloco C, $2^{\circ}$ piso, Florianópolis-SC, Brasil. CEP: 88040-900.

E-mail: brigido.camargo@yahoo.com.br.
} 
One hundred and fifty people participated in this research, divided into 3 age groups. In both analyzes there were no significant differences between respondents of different ages and all respondents seem to agree with the new way of thinking about the environment, recognizing its fragility and the need to care for it.

Keywords: Social representations. Environment. Social psychology.

\section{RESUMEN}

Desde hace algunas décadas el modo de pensar el medio ambiente ha sufrido cambios. La opinión de que el medio ambiente servía para satisfacer las necesidades humanas dio paso a la idea que los seres humanos somos parte de él y que él debe ser cuidado. Este estudio trata de identificar las actitudes $\mathrm{y}$ representaciones sociales del entorno de las personas de diferentes edades. Para eso se utilizó la escala NEP (nuevo paradigma ambiental) que mide las actitudes hacia el medio ambiente. También investigó las representaciones sociales de los encuestados sobre el medio ambiente, mediante la realización de un análisis de contraste mediante el programa Alceste. Los participantes fueron 150 personas, divididas en 3 grupos de edad. No se encontraron diferencias significativas entre los encuestados de diferentes edades y todos los encuestados parecen estar de acuerdo con la nueva forma de pensar sobre el medio ambiente, reconociendo su fragilidad y la necesidad de cuidar que él requiere.

Palabras clave: Representaciones sociales. Medio ambiente. Psicología social.

\section{INTRODUÇÃO}

O $s$ problemas relacionados com o meio ambiente e o cuidado ambiental têm despertado crescente interesse entre a população em geral e também na esfera acadêmica e científica. Isso vem ocorrendo porque, cada vez mais, o impacto humano sobre o meio ambiente tem resultado em desperdício e degradação dos recursos naturais. Considerando o impacto do comportamento humano sobre o cuidado ou a degradação ambiental e com o objetivo de compreender a relação que existe entre as pessoas e o ambiente, a Psicologia voltou seus esforços para essa área de interesse. Mas não se pode dizer que é atual o interesse da Psicologia pelos problemas ambientais, já que os primeiros estudos que buscam compreender a relação entre pessoa e ambiente datam da década de 1930 (Pol, 1993; Steg, Berg, \& Groot, 2012). 
Notadamente a Psicologia não é a única ciência a interessar-se por essa relação, tanto que a Psicologia ambiental tem origem fora do campo de estudos psicológicos, sofrendo influência da Arquitetura, da Geografia, da Biologia, entre outras (Giuliani \& Scopelliti, 2009; Pinheiro, 2003). Mas a especificidade da Psicologia está em seu interesse pela relação que as pessoas estabelecem com o ambiente, a qual é considerada bidirecional. Ao mesmo tempo em que a pessoa atua sobre o ambiente ao seu redor, o ambiente atua sobre a pessoa, exercendo influência sobre seus comportamentos, modos de pensar, crenças, valores, etc. (Polli \& Kuhnen, 2011).

Entretanto o modo como a pessoa representa o ambiente não é mediado apenas pela sua experiência direta, mas sofre influência das relaçôes sociais e do pensamento social dominante. A teoria das representações sociais é eficaz em explicar como o pensamento coletivo, ou o conhecimento do senso comum, tem origem, organiza-se e sofre mudanças na sociedade (Jodelet, 2001). A maneira como as pessoas representam o ambiente é vital para compreender relação pessoaambiente (Polli \& Kuhnen, 2011).

Para compreender essa forma de pensar o ambiente que é estabelecida na relação das pessoas com o entorno e que sofre influência das relações sociais, a teoria das representaçôes sociais surge como um aporte teórico adequado. Uma representação social é "uma forma de conhecimento socialmente elaborada e partilhada, com um objetivo prático, e que contribui para a construção de uma realidade comum a um conjunto social" (Jodelet, 2001, p.22).

Representações sociais são fenômenos da vida cotidiana. Quando as pessoas pensam sobre fatos relevantes, tomam partido, expressam e compartilham pensamentos por meio da comunicação, estão criando ou reproduzindo representações. As representações sociais, além de se configurarem como o pensamento compartilhado socialmente sobre um fato social que tem importância para um grupo de pessoas, também são teorizadas. A teoria das representaçõos sociais busca explicar e entender como ocorre o processo de criação do pensamento social, como ele se mantém ou se modifica, entre outros fatores (Polli, 2012).

O surgimento da teoria das representações sociais se deu na Europa, dentro do campo da Psicologia Social, com a publicação de La Psychanalyse: son image et son publique, por Serge Moscovici, em 1961, na França. No Brasil, foi traduzido parcialmente em 1978, com o título $A$ representação social da psicanálise. Em 2012, o texto original de Serge Moscovici foi traduzido integralmente para o português e recebeu o título de $A$ psicanálise, sua imagem e seu público, como na versão original em francês (Moscovici, 2012). Nesse estudo, Moscovici buscou 
compreender a representação social da psicanálise da população parisiense e teve como objetivo redefinir problemas e conceitos da Psicologia social a partir do fenômeno das representações sociais (Moscovici, 2012). A teoria das representações sociais surgiu com o interesse de compreender como o pensamento coletivo é organizado, assim como o próprio conteúdo do pensamento compartilhado socialmente.

Buscando ilustrar a composição das representações, Moscovici (2012) aponta três dimensões que as compõem: a informação, o campo de representação ou imagem e a atitude. A informação se refere ao conhecimento que um grupo de pessoas tem sobre um determinado objeto social, o objeto da representação. Somente é possível que essa dimensão exista se o grupo de fato tiver algum conhecimento sobre o objeto, pois é necessário que haja um saber consistente sobre ele. O campo de representação é a imagem, o modelo concreto que se relaciona a um aspecto preciso da representação social. Possibilita que normas possam ser criadas e seguidas, pois estabelece os limites da representação. A dimensão atitude se relaciona com a orientação global para a ação que a representação proporciona. Ela determina uma predisposição para agir em relação ao objeto da representação social. Pela identificação dos três componentes da representação, torna-se possível conhecer seu conteúdo e também o sentido dado ao objeto pelo grupo (Moscovici, 2012).

As representações sociais são relativamente estáveis em determinado grupo social, mas podem sofrer modificações ao longo do tempo. De modo que as representações sociais atuais sobre o meio ambiente podem diferir daquelas dominantes há algumas décadas. Essa hipótese é considerada com base na mudança ocorrida no pensamento social dominante sobre o meio ambiente ocorrida desde a década de 1960 até o momento atual. A ideia de que o meio ambiente estava à disposição do homem para atender a suas necessidades, aos poucos, foi dando lugar a uma nova forma de pensar relação humana com o meio ambiente. Atualmente a ideia de que as pessoas são parte do ambiente e que devem integrar-se a ele, pelo cuidado e bom uso dos recursos naturais, é predominante na sociedade (Polli, 2012).

Dos meados da década de 1960 até o momento atual, houve uma mudança de paradigma em relação ao meio ambiente. Historicamente, o homem tem-se servido da natureza para atender a suas necessidades de sobrevivência. Mas foi com a Revolução Industrial que as interações das pessoas com o meio ambiente passaram a ser caracterizadas pela busca de ganhos socioeconômicos (Coelho, Gouveia, \& Milfont, 2006). 
A partir da década de 1970, uma nova visão do meio ambiente vem ganhando espaço na sociedade, embora, até então, preponderassem crenças de que a natureza existe para suprir as necessidades humanas (Silva-Filho, Tochetto, Gravonski,Torres, \& Araújo 2007). Essas crenças compõem o chamado "paradigma social dominante" human exemptionalism paradigm (HEP), ou visão antropocêntrica, em que o meio ambiente ou a natureza é vista como um aglutinado de elementos físicos inertes. Ela pode e deve ser controlada pelos seres humanos, e serve para possibilitar ganhos econômicos. O progresso é considerado necessário, independentemente dos usos dos elementos naturais. Tal paradigma liga-se a crenças de crescimento, prosperidade, abundância e progresso. $\mathrm{O}$ homem não seria parte do meio ambiente, mas teria a função de dominá-lo (Mezzomo \& Nascimento-Schulze, 2004).

O próprio desenvolvimento econômico e industrial colocou em pauta uma crescente preocupação ambiental que originou mudanças nas crenças sobre a natureza e o meio ambiente. Um novo paradigma assentado na visão de que os recursos naturais devem ser cuidados, preservados, usados com critério vem ganhando lugar. $\mathrm{O}$ novo paradigma ambiental new environmental paradigm (NEP), ou visão ecológica (Dunlap \& Van Liére, 1978; Dunlap, Van Liére, Mertig, \& Jones, 2000), aos poucos, vem substituindo o paradigma até então dominante (Lima, 2006).

O novo paradigma abre caminho para o reconhecimento de que a humanidade pode enfrentar, ou vem enfrentando, diversos problemas relacionados à interdependência pessoa-ambiente, pois se pauta na ideia de inter-relação entre as sociedades humanas e o meio físico e natural, reconhecendo o ser humano como parte da natureza e não como um ser que se sobrepõe a ela. $\mathrm{O}$ novo paradigma ambiental traz à tona a noção de sustentabilidade, com o uso dos recursos naturais sem seu esgotamento, e coloca em voga a renovação dos recursos utilizados (Lima, 2006; Mezzomo \& Nascimento-Schulze, 2004; Sanz \& Guillén, 2005).

Ao analisar a transição paradigmática sob a ótica da Psicologia social, ainda mais precisamente sob o olhar da teoria das representações sociais, Castro (2003) destaca um aspecto bastante importante desse processo de mudança. Não se pode considerar o processo de mudança de paradigma como uma simples substituição de ideias, em que as velhas ideias antropocêntricas dão lugar às novas ideias ecológicas.

A teoria das representaçôes sociais possibilita que o processo de mudança seja identificado em termos de coexistência de ideias e não apenas em termos de substituição. Crenças profundamente arraigadas coexistem com novas crenças, 
e o processo de substituição do velho pelo novo é bastante lento, e, em certos casos, algumas crenças antigas podem manter-se indeterminadamente, apesar de novas crenças assumirem algum espaço (Castro, 2003).

Nesse sentido, a faixa etária pode ser importante na determinação das crenças que coexistem sobre o meio ambiente para cada pessoa. Pessoas de faixa etária mais elevada foram mais expostas ao paradigma social dominante, enquanto pessoas mais jovens foram expostas ao novo paradigma ambiental desde cedo. É importante identificar se as crenças diferem de acordo com a faixa etária ou se a sobreposição de ideias do novo paradigma ambiental vem ocorrendo com pessoas das mais variadas idades.

Este estudo, portanto, teve como objetivo identificar crenças de pessoas de diferentes faixas etárias sobre o meio ambiente, bem como identificar se suas atitudes diante do meio ambiente estão de acordo com o novo paradigma ambiental (NEP) ou o Paradigma Social Dominante (HEP).

\section{MÉTODO}

\subsection{Participantes}

Responderam aos instrumentos de coleta de dados 25 mulheres e 25 homens com idade entre 18 e 29 anos (jovens), 25 mulheres e 25 homens com idade entre 30 e 59 anos (adultos), 25 mulheres e 25 homens com 60 anos ou mais (mais velhos). Todos os respondentes foram contatados nas dependências do Serviço Social do Comércio (Sesc), unidade do Estreito, Florianópolis, Santa Catarina. O local foi escolhido por possibilitar uma equivalência no ambiente sociocultural independentemente da faixa etária dos participantes.

\subsection{Instrumento}

A coleta de dados foi realizada em dois momentos distintos, e os instrumentos de ambas as etapas continham questões que não serão tratadas neste artigo. $\mathrm{Na}$ primeira etapa de coleta, os participantes responderam a uma questão aberta em que deveriam descrever as informaçôes que tinham sobre o meio ambiente.

$\mathrm{Na}$ segunda etapa, os participantes responderam a uma escala de atitude em relação ao meio ambiente (escala NEP), em que 14 afirmações sobre o tema foram apresentadas e os respondentes deveriam responder desde concordo totalmente até discordo totalmente, em uma escala de 5 pontos, em que o ponto médio foi nem concordo nem discordo, e havia uma opção para marcação da resposta não sei, que foi contabilizada como não resposta. Em ambas as etapas, ao final do questionário, havia questôes para identificação do perfil socioeconômico. 


\subsection{Procedimento}

Os participantes foram abordados durante a realização de atividades variadas nas dependências do Sesc-SC. Todos foram convidados a participar e esclarecidos sobre a não obrigatoriedade de participação e sigilo sobre os dados fornecidos. Assinaram ao termo de consentimento livre e esclarecido e responderam ao questionário de forma escrita. As pessoas que tinham alguma dificuldade para ler ou escrever responderam à pesquisadora em forma de entrevista. As respostas foram anotadas e transcritas de modo semelhante ao procedimento adotado com os demais participantes.

\subsection{Análise de dados}

Os dados oriundos da escala NEP foram tratados estatisticamente em termos de média e desvio padrão. Foi realizada uma análise de variância simples (Anova), que possibilitou que fossem identificados os principais fatores relacionados às crenças compatíveis com o HEP e crenças compatíveis com o NEP.

A análise dos dados obtidos pelas questões abertas foi lexical, para tanto, foi utilizado o programa Alceste (Analyse Lexicale par Contexte d'un Ensemble de Segments de Texte) (Camargo, 2005). Foi realizada uma análise por contraste, utilizando o programa para identificar as particularidades de cada faixa etária.

\section{RESULTADOS}

\subsection{Atitude em relação ao meio ambiente}

Foi solicitado aos participantes que respondessem à escala de atitude em relação ao meio ambiente NEP, que mede as crenças ante o meio ambiente que podem ser compatíveis com o HEP ou com o NEP. A escala é composta por 14 itens, divididos em 5 dimensões:

a) limites de crescimento;

b) antiantropocentrismo;

c) fragilidade do equilíbrio da natureza;

d) rejeição ao antigo paradigma; e

e) possibilidade de crise ecológica. Cada uma das dimensões é composta por 3 itens, exceto a dimensão 1, que é composta por 2 itens. 
Foi calculada a confiabilidade da escala, e o teste apresentou um alfa de Cronbach de valor 0,63, o que indica que a confiabilidade da escala é apenas razoável, pois, para apresentar uma confiabilidade ótima, o valor deveria ser igual ou superior a 0,75 . A média geral da escala foi $1,98(\mathrm{DP}=0,50)$, abaixo do ponto médio da escala $(3,00)$, o que indica que atitude geral dos respondentes em relação ao meio ambiente é compatível com o NEP.

Tabela 1. Médias e desvios padrões por dimensões da NEP em relação à faixa etária

\begin{tabular}{|l|l|l|l|l|l|l|c|}
\hline & \multicolumn{7}{|c|}{ Dimensões } \\
\hline $\begin{array}{l}\text { Faixa } \\
\text { etária }\end{array}$ & & $\mathbf{1}$ & $\mathbf{2}$ & $\mathbf{3}$ & $\mathbf{4}$ & $\mathbf{5}$ & $\begin{array}{c}\text { Escore } \\
\text { geral }\end{array}$ \\
\hline \multirow{2}{*}{$\mathbf{2}$} & $\mathrm{M}$ & 2,27 & 1,82 & 1,95 & 2,26 & 1,68 & 1,96 \\
\hline \multirow{2}{*}{} & DP & 0,92 & 0,70 & 0,75 & 0,77 & 0,67 & 0,46 \\
\hline \multirow{3}{*}{$\mathbf{3}$} & M & 2,11 & 1,85 & 1,81 & 2,33 & 1,50 & 1,90 \\
\hline \multirow{2}{*}{ Geral } & DP & 1,08 & 0,73 & 0,71 & 0,88 & 0,57 & 0,50 \\
\hline & M & 2,24 & 2,19 & 1,74 & 2,69 & 1,49 & 2,06 \\
& DP & 1,29 & 0,91 & 0,91 & 0,94 & 0,72 & 0,54 \\
\hline
\end{tabular}

Fonte: elaborada pelos autores.

Pela análise realizada por dimensões da escala, não foram encontradas diferenças entre as faixas etárias, sendo que todas as faixas apresentaram médias abaixo do ponto médio da escala em todas as dimensões, o que demonstra que as crenças dos participantes da pesquisa são mais compatíveis com o novo paradigma ambiental (NEP) independentemente da idade.

Optou-se, portanto, por realizar uma análise item a item da escala para identificar possíveis diferenças entre os respondentes das três faixas etárias: jovens, adultos e mais velhos. A análise de variância de um fator (Anova) indicou diferenças estatisticamente significativas entre os grupos em relação ao item 3, Quando as pessoas interferem na natureza produzem quase sempre consequências desastrosas $[\mathrm{F}(2,128)=5,36, \mathrm{p}<0,05]$; item 4 , A engenhosidade humana garantirá que nós não deixemos a terra sem condiçôes de vida $[\mathrm{F}(2,133)=5,64, \mathrm{p}<0,05]$; e item 7, As plantas e os animais têm tanto direito quanto as pessoas de existir $[\mathrm{F}(2,141)=3,65, \mathrm{p}<0,05]$. 
O pós-teste de Tukey (DHS) indicou as diferenças significativas grupo a grupo e a força de tais relações. Em relação ao item 3, Quando as pessoas interferem na natureza produzem quase sempre consequências desastrosas, houve diferença significativa $(\mathrm{p}<0,05)$ entre os respondentes jovens e mais velhos e entre jovens e adultos, sendo que os jovens demonstraram estar parcialmente de acordo com a afirmação $(m=2,11)$, enquanto os respondentes adultos e mais velhos demonstraram estar de acordo ( $\mathrm{m}=1,54$ e 1,45 , respectivamente). A diferença entre os grupos pode ser considerada forte tanto na comparação dos jovens com os mais velhos $(\mathrm{d}=0,62)$, como $(\mathrm{d}=0,54)$.

Em relação ao item 4 da escala, $A$ engenhosidade humana garantirá que nós não deixemos a terra sem condiçôes de vida, foram identificadas diferenças significativas entre os respondentes jovens e mais velhos $(\mathrm{p}<0,05)$. Os jovens concordam com a afirmação $(m=2,46)$, enquanto os mais velhos discordaram $(m=3,45)$, a diferença entre os dois grupos é considerada forte $(\mathrm{d}=0,71)$.

$\mathrm{O}$ item $7 \mathrm{da}$ escala, As plantas e os animais têm tanto direito quanto as pessoas de existir, apresentou diferenças significativas de resposta entre os grupos etários extremos $(\mathrm{p}<0,05)$, a saber: jovens e mais velhos, embora ambos os grupos concordem com a afirmação, a força da relação pode ser considerada média ( $d$ $=0,44)$.

Embora algumas diferenças pontuais tenham sido encontradas entre os respondentes das três faixas etárias, os resultados encontrados demonstram que a hipótese inicial de que as pessoas com mais idade poderiam apresentar atitudes compatíveis com o paradigma social dominante, por terem sido expostas e ele durante parte da sua formação educacional, não se confirmou. Apesar de terem sido expostas a tais crenças ao longo de seu desenvolvimento, as informações atuais se mostram incorporadas ao modo de pensar de todas as pessoas, independentemente de seu contato anterior com o paradigma social dominante, de modo que o novo paradigma ambiental parece ganhar força entre todos os participantes da pesquisa.

\subsection{Análise de contraste}

Para identificar possíveis diferenças entre as informações que pessoas de faixas etárias distintas têm sobre o meio ambiente, foi realizada uma análise de contrate com o auxílio do programa Alceste. Nessa etapa de análise, o conteúdo produzido em resposta à questão aberta sobre as informações mais importantes que os respondentes tinham sobre o meio ambiente os mesmos grupos etários foram comparados. 
Verificou-se que, entre as 149 unidades de contexto iniciais ou respostas (UCEs), 50 foram enunciadas pelos jovens, 50 pelos adultos e 49 pelos participantes mais velhos. A tabela 2 ilustra as palavras com maior associação a cada uma das faixas etárias, mostrando as principais diferenças dos conteúdos expressos por respondentes das diferentes faixas etárias.

Tabela 2. Resultados da análise de contraste por faixa etária

\begin{tabular}{|c|c|c|c|c|c|c|c|c|}
\hline \multicolumn{3}{|c|}{ Faixa 1 } & \multicolumn{3}{c|}{ Faixa 2 } & \multicolumn{3}{c|}{ Faixa 3 } \\
\hline Palavra & Freq. & $\mathbf{c}^{2}$ & Palavra & Freq. & $\mathbf{c}^{2}$ & Palavra & Freq. & $\mathbf{c}^{2}$ \\
\hline Meio & 13 & 5 & Planeta & 11 & 4 & Pessoa & 12 & 5 \\
\hline Vivem & 12 & 4 & Depende & 6 & 5 & Lixo & 11 & 4 \\
\hline Ele & 11 & 8 & Espécie & 4 & 5 & Rio & 11 & 10 \\
\hline Animais & 11 & 4 & Ecossistema & 4 & 8 & Mata & 7 & 7 \\
\hline Cada & 10 & 8 & Continuar & 4 & 6 & Floresta & 7 & 4 \\
\hline Onde & 10 & 7 & & & & Sabe & 5 & 7 \\
\hline Equilíbrio & 10 & 7 & & & & Saúde & 5 & 5 \\
\hline Homem & 9 & 4 & & & & Dever & 4 & 5 \\
\hline Humano & 9 & 4 & & & & Proteção & 4 & 5 \\
\hline Dia & 8 & 5 & & & & Derrubada & 4 & 8 \\
\hline Recursos & 7 & 7 & & & & Mar & 4 & 6 \\
\hline Vez & 6 & 9 & & & & & & \\
\hline Pode & 5 & 7 & & & & & & \\
\hline Desastres & 4 & 8 & & & & & & \\
\hline Maneira & 4 & 6 & & & & & & \\
\hline Extinção & 4 & 5 & & & & & \\
\hline
\end{tabular}

Fonte: elaborada pelos autores.

Para os jovens, o meio ambiente é local onde habitamos e dividimos este habitat com outras formas de vida, animal e vegetal. Eles parecem perceber que os seres humanos são parte do meio ambiente, sentem-se integrantes. Demonstram ainda preocupações com seu cuidado, demonstrando estar cientes do mal que os seres humanos causam ao equilíbrio ambiental. Alguns trechos de respostas ilustram essa forma de conhecimento: 
É o ambiente que vivemos que a cada dia está ficando degradado com a poluição, destruição e com os desmatamentos das nossas reservas florestais. Com isso, cada vez mais estamos tendo desastres naturais.

O meio ambiente é, como o próprio nome sugere, o espaço em que vivemos. Ele engloba diferentes grupos, pessoas, animais, vegetais, etc., bem como a infraestrutura utilizada por esses grupos. Atualmente se encontra ameaçado em virtude dos excessos cometidos pelo ser humano.

Os adultos destacam os conhecimentos relacionados ao cuidado com o meio ambiente e também com a sua importância para a vida. Reconhecem ainda a responsabilidade humana em seu cuidado. Algumas respostas ilustram o tipo de conhecimento enunciado pelos respondentes dessa faixa etária:

O meio ambiente é a nossa casa, o planeta todos os elementos da natureza. Nós, seres humanos, somos responsáveis por sua preservação. Dele depende nosso bem-estar e saúde.

Sei que é extremamente importante a preservação, pois disso depende a sobrevivência da espécie humana e outras.

Já para os mais velhos se destacam os conhecimentos relacionados aos problemas ambientais, alguns problemas são elencados e as pessoas são responsabilizadas. Eles apontam para a necessidade de cuidado. Alguns trechos das respostas são ilustrativos:

Queimadas, derrubada de árvores na Amazônia, devastação, cortam sem reflorestar.

Despoluiçãa dos rios e dos oceanos é importante. É muito importante que as pessoas se conscientizem que deverão ajudar para proteção do meio ambiente. As grades queimadas, a falta de mata ciliar que é muito importante. O acúmulo de lixo sem cuidado é um poluente terrivel.

A análise de contrataste permitiu identificar diferenças na dimensão informacional da representação social do meio ambiente em pessoas de faixas etárias distintas. Para os respondentes de faixa etária mais jovem, destaca-se o pertencimento que sentem em relação ao meio ambiente. As pessoas de faixa etária intermediária (adultos) demonstram conhecimentos mais dispersos, e destaca-se o conhecimento de que o meio ambiente é o lugar em que a vida pode existir. Já os respondentes com idade mais elevada destacam os problemas 
ambientais. Tais diferenças ilustram as individualidades, as especificidades da representação social do meio ambiente para faixas etárias distintas.

Nota-se também que todos os grupos etários reconhecem a importância com o cuidado ambiental, seja para manutenção da vida humana ou animal ou para evitar desastres futuros. A responsabilização das pessoas pelos problemas relacionados ao meio ambiente também permeia o discurso de todas as faixas etárias, evidenciando o caráter consensual da representação social do meio ambiente.

Os dados encontrados através da análise de contraste, realizada com o auxílio do programa Alceste, parecem apresentar sincronia com os achados da escala NEP.

\section{DISCUSSÃO}

Os resultados encontrados neste estudo indicam que, independentemente da faixa etária dos respondentes, o seu pensamento sobre o meio ambiente parece ser compatível com o novo paradigma ambiental, que considera os seres humanos como parte de uma natureza que é frágil e precisa de cuidados. Tais resultados diferem daqueles encontrados por Castro (2003) em Portugal, pois a autora encontrou diferentes formas de pensar o meio ambiente.

Ao procurar identificar as representações sociais do ambiente e da natureza Castro (2003) verificou que a representação social era composta por ideias ecológicas de que a terra tem limitações de espaço e de recursos, e que as pessoas estão sujeitas a tais limites, mas que têm utilizado os recursos naturais sem critério e, portanto, comprometem o equilíbrio natural. Por outro lado, encontrou-se uma ideia diversificada, antropocêntrica, em que as pessoas acreditam que os seres humanos são capazes de domar a natureza e a ciência é capaz de fornecer explicaçôes para os fatos. A autora verificou que a ideia ecológica predominava em relação à ideia antropocêntrica, indicando que a visão da natureza e do ambiente como elementos que precisam ser cuidados pelos seres humanos vem se tornando uma normativa e ganha espaço no discurso popular.

É preciso ter cuidado ao comparar os achados deste estudo com os achados de Castro em Portugal, pois os dois estudos apresentam diferenças tanto em relação aos participantes como também em relação ao ambiente sociocultural em que eles estão inseridos. É ainda necessário ter em conta que a autora não trabalhou com comparação entre pessoas de diferentes faixas etárias. De tal modo que algumas hipóteses podem ser lançadas na busca da compreensão das diferenças encontradas. 
O estudo realizado por Castro data de 2003, e a autora identificou que as crenças compatíveis com o novo paradigma ambiental estavam mais presentes do que as crenças compatíveis com o paradigma antropocêntrico. Pode-se deduzir que, com o passar de alguns anos, as crenças antigas tenham dado lugar às novas crenças. Outro fator de interferência é o fator cultural, pois a realidade ambiental de Portugal é diferente da realidade brasileira, fato que pode impactar sobre o modo de pensar dos respondentes sobre a temática.

Cabe ainda considerar que a homogeneidade encontrada entre os respondentes de diferentes faixas etária neste estudo pode ser devido às semelhanças no ambiente sociocultural dos participantes do estudo.

\section{CONSIDERAÇÕES FINAIS}

Os achados deste estudo não podem ser comparados com estudos de cunho semelhante realizados no Brasil, já que, até o momento, a literatura carece de estudos que busquem identificar as atitudes das pessoas em relação ao meio ambiente por meio da utilização da escala NEP. Ainda que essa escala não venha sendo utilizada em muitos estudos no Brasil, seus resultados se mostram promissores. No entanto é importante aprimorar a escala de modo a melhorar sua consistência interna. Cabe ainda considerar que o valor baixo do alfa de cronbach $(0,63)$ possa ser devido ao pequeno número de itens da escala $(14)$.

Como forma de preencher esta lacuna do conhecimento, este estudo se vale da teoria das representaçóes sociais e utiliza a análise de contrates para confirmar os achados obtidos pela utilização da escala NEP. As duas análises confirmam que o pensamento dos respondentes parece ser compatível com o novo paradigma ambiental. Tais achados se mostram promissores, já que o novo paradigma ambiental se refere ao sentimento de pertencimento em relação ao meio ambiente, que traz consigo a preocupação com o cuidado ambiental e o reconhecimento da fragilidade do meio ambiente. 


\section{REFERÊNCIAS}

Camargo, B. V. (2005). Alceste: um programa informático de análise quantitativa de dados textuais. In A. S. P. Moreira, B. V. Camargo, J. C. Jesuíno \& M. N. Sheva (Eds.), Perspectivas teórico-metodológicas em representaçôes sociais (pp. 511-539). João Pessoa: Editora Universitária UFPB.

Castro, P. (2003). Pensar a natureza e o ambiente: alguns contributos a partir da Teoria das Representaçôes Sociais. Estudos de Psicologia, Natal, 8, 263-271.

Coelho, J. A. P. M., Gouveia, V. V., \& Milfont, T. L. (2006). Valores humanos como explicadores de atitudes ambientais e intenção de comportamento próambiental. Psicologia em estudo, Maringá, 1(11), 199-207.

Dunlap, R. E., \& Van Liére, K. D. (1978). The new environmental paradigm: a proposed mesuring instrument and preliminary results. Journalof Environmental Education, 9(4), 10-19.

Dunlap, R. E., Van Liére, K. D., Mertig, A. G., \& Jones, R. E. (2000). Measuring Endorsement of the New Ecological Paradigm: A Revised NEP Scale - Statistical Data Included. Journal of social issues, 56(3), 425-442.

Giuliani, M. V., \& Scopelliti, M. (2009). Empirical research in environmental psychology: Past, present, and future. Journal of Environmental Psychology, 375-386.

Jodelet, D. (2001). Representações sociais: um domínio em expansão. In D. Jodelet (Ed.), As representaçôes sociais (pp. 17-44). Rio de Janeiro: EdUERJ.

Lima, A. V. (2006). Os portugueses e o ambiente: viver a natureza, pensar o desenvolvimento. In Anais, 8 curso de verão da Ericeira, Ericeira: ICEA.

Mezzomo, J., \& Nascimento-Schulze, C. M. (2004). O impacto de uma exposição científica nas representaçôes sociais sobre meio ambiente: um estudo com alunos do ensino médio. Comunicação e Sociedade, 6, 151-170.

Moscovici, S. (2012). A Psicanálise, sua imagem e seu público. Petrópolis: Vozes.

Pinheiro, J. Q. (2003). Psicologia ambiental brasileira no início do século XXI: sustentável? In O. H. Yamamoto \& V. V. Gouveia (Eds.), Construindo a psicologia brasileira: desafios da ciência e prática psicológica (pp. 279-313). São Paulo: Casa do Psicólogo. 
Pol, E. (1993). Environmental psychology um Europe from architectural psychology to green psychology. Aldershot: Avebury.

Polli, G. M. (2012). Representaçôes sociais do meio ambiente e da água na mudança de paradigmas ambientais. (Tese de Doutorado). Universidade Federal de Santa Catarina, Programa de Pós-Graduação em Psicologia. Florianópolis.

Polli, G. M., \& Kuhnen, A. (2011). Possibilidades de uso da teoria das representações sociais para os estudos pessoa-ambiente. Estudos de Psicologia, Natal, 16, 57-64.

Sanz, L. V., \& Guillén, C. S. J. (2005). Escala nuevo paradigma ecológico: propiedades psicométricas con uma muestra española obtenida a través de internet. Médio ambiente y comportamiento humano, 6(1), 37-49.

Silva-Filho, J. C. L., Tochetto, M. R. L., Gravonski, I., Torres, F.S. \& Araújo, N.R.S. (janeiro/abril 2009). Análise comparativa do novo paradigma ecológico em dois estados brasileiros: a gestão ambiental além do mercado e do estado. Revista Gestão.Org. 7(1), 84-101. Recuperado a partir de http://www.revista.ufpe.br/gestaoorg/index.php/gestao/article/view/100/89.

Steg, L., Berg, A. E. V. D., \& Groot, J. I. M. d. (2012). Environmental psychology: an introduction. Oxford: BPS Wiley-Blackwell. 